\title{
POEMS Syndrome Presenting with Fever, Diarrhea and Progressive Fatigue:
}

\section{A Case Report}

\section{Fang Chen | Heng Jin | Song-Tao Shou*}

*Correspondence: Song-Tao Shou

Address: Department of Emergency Medicine, Tianjin Medical University General Hospital, Tianjin 300052, China

e-mail $\bowtie$ : zyyshou@tmu.edu.cn

Received: 30 April 2021; Accepted: 05 May 2021

Copyright: (C) 2021 Chen F. This is an open-access article distributed under the terms of the Creative Commons Attribution License, which permits unrestricted use, distribution, and reproduction in any medium, provided that the original work is properly cited.

\section{ABSTRACT}

We report a successful diagnosis of POEMS syndrome (Polyneuropathy, Organomegaly, Endocrinopathy, Monoclonal protein, Skin changes) patient who presented with fever, diarrhea and progressive fatigue in the lower limbs. The aim of this case report is to enhance the awareness of POEMS syndrome. When clinicians encounter a patient with unexplained peripheral neuropathy or multisystem disorders, and also suffers from diabetes mellitus or other endocrinopathy, detailed examinations should be performed to rule out POEMS syndrome.

Keywords: POEMS Syndrome, Fatigue, VEGF, Polyneuropathy

\section{Introduction}

POEMS syndrome is a rare multi-system damaging disease associated with plasma cell disorder, which was coined by Bardwick in 1980 (Bardwick et al., 1980). A national survey conducted in Japan in 2003 indicated that the incidence rate was 0.003\% (Nasu et al., 2012). A literature-based study in China shows that, the percentage of misdiagnosis was 85\% (Wang et al., 2019), thus its incidence actually may be higher. The median survival time was only 5-7 years if without successful treatment, and common causes of death include cardiopulmonary failure, coagulation disorders, long-term use of glucocorticoids and infections caused by immunosuppressive agents (Wang et al., 2019). Therefore, early identification of this disease is very important. We report a successful diagnosis of POEMS syndrome patient who presented with fever, diarrhea and progressive fatigue in the lower limbs.

\section{Case Presentation}

On August 12th, 2020, a 52-year-old male patient was admitted to emergency department of Tianjin Medical University General Hospital, complaining of fever, diarrhea for two weeks and progressive limbs weakness and incapablity to stand for a day. 
Two weeks ago, the patient had a fever every afternoon, the highest temperature was $37.6^{\circ} \mathrm{C}$, and accompanied by diarrhea, stool $4 \sim 5$ times per day, and with progressive weakness. No other accompanied symptoms. Based on the physical examinations and laboratory test, the patient was diagnosed as bacterial infection and treated with levofloxacin and infusion. After remmision of the fever and diarrhea, he developed progressive fatigue and numbness in lower limbs. More serious is the inability to stand.

The patient's left femoral fracture was fixed with steel nail for more than 20 years, no coronary heart disease, hypertension, diabetes mellitus, alcohol consumption, smoking and drug abuse.

On admission, temperature was $37.1^{\circ} \mathrm{C}$, pulse rate, $93 \mathrm{bpm}$, respiration rate, $18 \mathrm{bpm}$, blood pressure, 120/77 mmHg, pulse oximetry saturation, 97\%. No abnormal breath sound in both lungs, Heart sounds were normal. Liver and spleen were not touched. There was no tenderness and rebound tenderness on abdomen. The lower limbs were not swollen, and muscle strength of limbs was grade 4.

Blood route was nomal. The patient's cardiac, liver, and renal function were normal. $\mathrm{K}^{+}, \mathrm{Na}^{+}$and $\mathrm{Ca}^{+}$were normal. Fasting blood glucose concentration was $7.4 \mathrm{mmol} / \mathrm{L}$, glycosylated hemoglobin concentration was $6.20 \%$. Oral glucose tolerance test revealed that he was suffering from type 2 diabetes mellitus. Lumbar puncture, cerebrospinal fluid routine was normal. Protein detection in cerebrospinal fluid was increased $2.58 \mathrm{~g} / \mathrm{L} \uparrow$. Cerebrospinal fluid examination indicated albuminocytologic dissociation. Abdominal ultrasound revealed splenomegaly.

The patient was initially diagnosed with weakness of unknown origin, Guillain-Barre syndrome or Myasthenia gravis. Infective fever, Diabetes. He was given a diagnostic therapy with vitamin B nutrition for neurotherapy (LIN et al., 2017) and methylprednisolone suppressive immunotherapy and gamma globulin shock therapy for three days (Yang Guangwei and Deng Nan, 2019). His body temperature was normal, but weakness of the lower limbs did not improve.

\section{Final Diagnosis}

Additional tests revealed that the patient was negative for acetylcholine receptor and musclespecific tyrosine kinase antibodies (Chang et al., 2020). Electromyography showed severe multiple peripheral neuropathy affecting both the lower limbs. Diagnosis of Guillain-Barre syndrome and myasthenia gravis was excluded (LIN et al., 2017). The patient's serum IgG level was positive with $\kappa$ light chain restriction. His serum $\lambda$ light chain concentration was $831 \mathrm{mg} / \mathrm{dL}$, and his light chain $\kappa$ concentration was $994 \mathrm{mg} / \mathrm{dL}$. He underwent bone puncture, and a bone marrow biopsy revealed granulocytosis and megakaryocyte hyperplasia, with many immature plasma cells (Fig. 1). Pathology of 
the bone marrow biopsy revealed clonal hyperplasia of plasma cells (Fig. 2). Vascular endothelial growth factor (VEGF) measurement was $>800 \mathrm{pg} / \mathrm{ml}$. Cervical and lumbar spine CT revealed polyosclerosis (Fig. 3 and Fig. 4).

The patient met both mandatory major criteria for POEMS syndrome (polyneuropathy, monoclonal plasma cell-proliferative disorder), two of the three other major criteria (sclerotic bone lesions, VEGF elevation) and two of the six minor criteria (splenomegaly, diabetes, skin changes) (Dispenzieri, 2019). The patient was diagnosed with POEMS syndrome.

\section{Treatment and Outcome}

He was treated with oral lenalidomide (25 mg/d), dexamethasone $20 \mathrm{mg} / \mathrm{d}$ BIW (The first two days of the week) (Nozza et al., 2017). One course of treatment lasts for three weeks. After two courses of treatment, the patient's muscle strength of the limbs had been corrected.

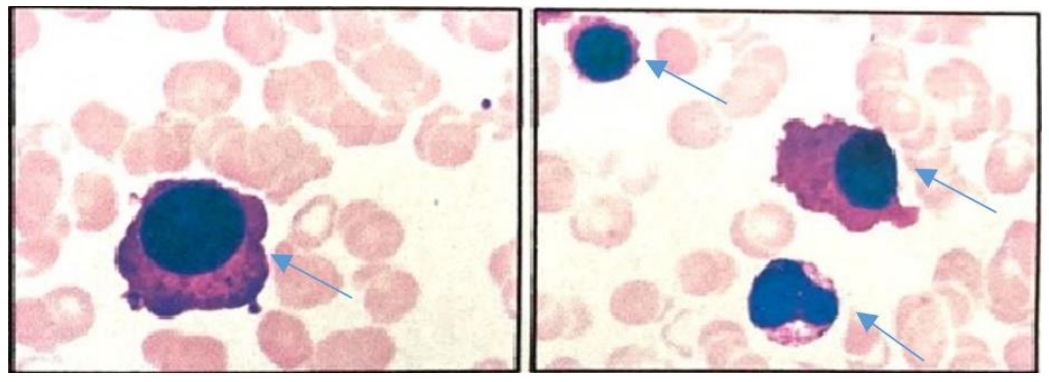

Figure 1: Bone marrow smear.
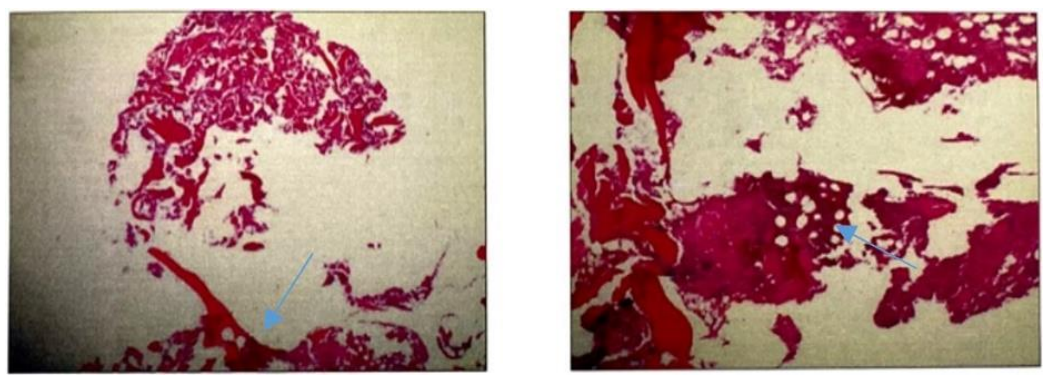

Figure 2: Pathological examination of bone marrow biopsy, the arrow indicates an increase in plasma cells.

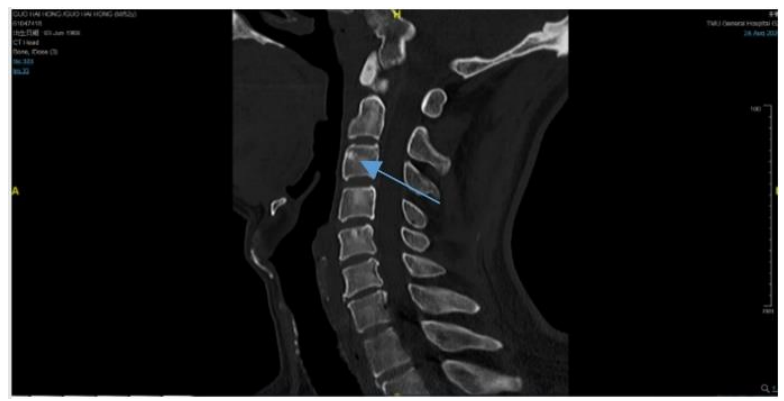

Figure 3: CT scan of the cervical spine. 

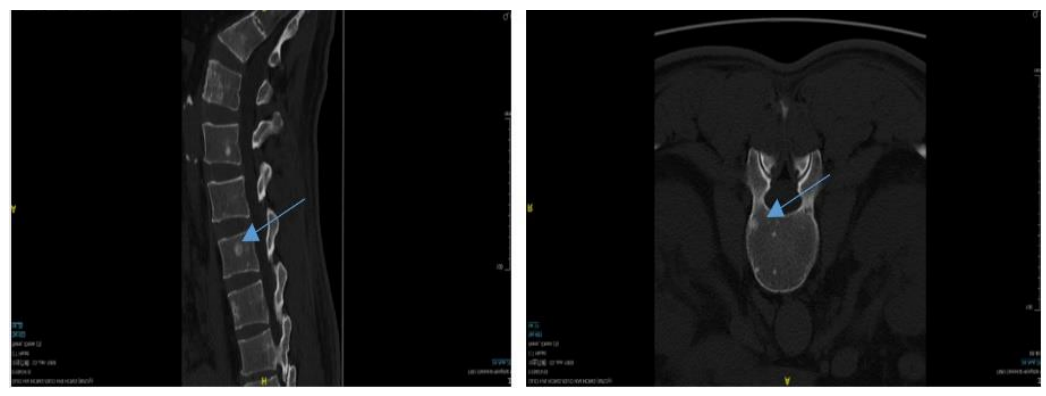

Figure 4: CT scan of the lumbar spine.

\section{Discussion}

POEMS syndrome is characterized by polyneuropathy, organomegaly, endocrinopathy, Mproteinemia (M-protein), and skin changes as the principal clinical manifestations. The paraneoplastic syndrome caused by abnormal proliferation of plasma cells which is termed by POEMS syndrome (Bardwick et al., 1980). In 2019, Dispenzieri updated the diagnostic criteria. The principal criteria are as follows: Polyneuropathy (typically demyelinating); Monoclonal plasma cell-proliferative disorder (usually of the $\lambda$ light chain); Castleman disease; Sclerotic bone lesions; Vascular endothelial growth factor elevation (Dispenzieri, 2019). Secondary criteria: Organomegaly (enlargement of the spleen, liver, or lymph nodes); Extravascular volume overload (edema, pleural effusion, or ascites); Endocrine disease (adrenal, thyroid, pituitary, gonadal, parathyroid, or pancreas); Skin changes (hyperpigmentation, hypertrichosis, glomeruloid hemangiomata, plethora, acrocyanosis, flushing, white nails); Papillary edema; Thrombocytosis or polycythemia. Diagnosis of POEMS syndrome requires 3 of the main criteria to be met, of which Polyneuropathy and Monoclonal plasma cell-proliferative disorder are mandatory, and at least one secondary criterion (Dispenzieri, 2019).

A number of researchers have conducted a comprehensive genetic and transcriptional analysis of plasma cells and found that clones have a unique role in the pathogenesis of POEMS syndrome (Kamezaki and Nagafuji, 2007). POEMS syndrome can be easily missed and misdiagnosed. Because of the clinical manifestations with the involvement of multiple organs, the primary clinical manifestations are diverse such as peritoneal effusion (Shi Xiaoyan et al., 2021), hepatosplenomegaly, numbness of the limbs, proteinuria, etc. In this case, the initial manifestation of POEMS syndrome was fever and diarrhea, after that he felt weakness in both limbs. Fever may be the predisposing factor of the disease. Although the patient had a diarrhea, there were no abnormalities in electrolytes and no signs of dehydration. After remmision of the fever and diarrhea, he developed progressive fatigue and numbness in limbs. Therefore, detailed examinations should be performed. Thus the patient underwent lumbar puncture, examination of the cerebrospinal fluid revealed protein-cell separation, with a coexisting fever and diarrhea, easily misdiagnosed as Guillain-Barré syndrome. However the electromyography, cervical and lumbar CT, bone 
puncture, immunofixation electrophoresis and Vascular endothelial growth factor (VEGF) measurement showed that he was more likely to be diagnosed with POEMS syndrome.

The pathogenesis of POEMS syndrome remains unclear. Patients are often diagnosed for the first time with polyneuropathy in clinical practice. A study has suggested that an increasing VEGF concentration is strongly related to POEMS syndrome, mainly the VEGF165-subtype8 (Dispenzieri, 2019). Few studies have demonstrated that in patients with POEMS syndrome, VEGF is abnormally elevated, observations that have a sensitivity of $100 \%$ and specificity of $91 \%$. Its overexpression can regulate endothelial cell proliferation, activate vascular smooth muscle cells, promote neovascularization, and cause vascular endothelial dysfunction (Pihan et al., 2018), disorders in VEGF may lead to ascites, edema, organomegaly, pleural effusion, and neuropathy (Wu Pingping et al., 2020). Thus, early measurement of VEGF levels could to a certain extent avoid misdiagnosis or missed diagnosis of POEMS syndrome (Marsh et al., 2021).

A study has shown that high-risk factors for POEMS syndrome are: age $>50$ years, pleural effusion, pulmonary hypertension, glomerular filtration rate $<30 \mathrm{ml} / \mathrm{min} / 1.73 \mathrm{~m}^{2}$, etc. (Wang et al., 2017). There is no standard of treatment for POEMS syndrome, but patients with small levels of sclerosing bone injury usually receive radiotherapy. Conversely, severely-affected patients can be treated with chemotherapy and hematopoietic stem cell transplantation (Ali and Qazilbash, 2021). In clinical practice, the study and appreciation of POEMS syndrome require strengthening, so as to achieve early diagnosis and treatment of the disease, and thereby improve prognosis.

\section{Conclusion}

The aim of this case report is to enhance the awareness of POEMS syndrome. At present, the underlying pathogenesis is still unknown. POEMS syndrome is easily misdiagnosed, such as GuillainBarre syndrome, diabetic peripheral neuropathy, and other similar diseases. Therefore, when clinicians encounter a patient with unexplained peripheral neuropathy or multisystem disorders, and also suffers from diabetes or other endocrinopathy, detailed examinations should be performed to rule out POEMS syndrome.

\section{References}

Ali T and Qazilbash MH. POEMS syndrome: A multisystem clonal disorder. Eur J Haematol 2021; 106: 14-18.

Bardwick PA, Zvaifler NJ, Gill GN, Newman D, Greenway GD, Resnick DL. Plasma cell dyscrasia with polyneuropathy, organomegaly, endocrinopathy, M protein, and skin changes: the POEMS syndrome. Report on two cases and a review of the literature. Medicine 1980; 59: 311-322. 
Chang T. Guidelines for diagnosis and treatment of myasthenia gravis in China 2020. Chinese Journal of Neuroimmunology and Neurology 2020; 201: 1-12.

Dispenzieri A. 'Poems Syndrome: 2019 Update on Diagnosis, Risk-Stratification, and Management'. Am J Hematol 2019; 94: 812-827.

Kamezaki K and Nagafuji K. POEMS syndrome: monoclonal plasma cell disorder with polyneuropathy. Nihon Rinsho 2007; 65: 2235-2237.

LIN Guowei, WANG Jiyao, GE Junbo. Practical Internal Medicine 15th Edition. Science Technology and Publishing House 2017: 2 .

Marsh ES, Keddie S, Terris-Prestholt F, D'Sa S, Lunn MP. Early VEGF testing in inflammatory neuropathy avoids POEMS syndrome misdiagnosis and associated costs. J Neurol Neurosurg Psychiatry 2021; 92: 172-176.

Nasu S, Misawa S, Sekiguchi Y, Shibuya K, Kanai K, Fujimaki Y, Ohmori S, Mitsuma S, Koga S, Kuwabara S. Different neurological and physiological profiles in POEMS syndrome and chronic inflammatory demyelinating polyneuropathy. $J$ Neurol Neurosurg Psychiatry 2012; 83: 476-479.

Nozza A, Terenghi F, Gallia F, Adami F, Briani C, Merlini G, Giordano L, Santoro A, Nobile-Orazio E. Lenalidomide and dexamethasone in patients with POEMS syndrome: results of a prospective, open-label trial. Br J Haematol 2017; 179 : 748-755.

Pihan M, Keddie S, D'Sa S, Church AJ, Yong KL, Reilly MM, Lunn MP. Raised VEGF: High sensitivity and specificity in the diagnosis of POEMS syndrome. Neurol Neuroimmunol Neuroinflamm 2018; 5: e486.

Shi Xiaoyan, Peng Hongju, Sun Guozhen. A case of POEMS syndrome with a large amount of ascites as the main manifestation and literature review. Journal of Shandong University 2021; 59: 114-117.

Wang C, Huang XF, Cai QQ, Cao XX, Duan MH, Cai H, Zhou DB, Li J. Prognostic study for overall survival in patients with newly diagnosed POEMS syndrome. Leukemia 2017; 31: 100-106.

Wang Y, Huang LB, Shi YH, Fu H, Xu Z, Zheng GQ, Wang Y. Characteristics of 1946 Cases of POEMS Syndrome in Chinese Subjects: A Literature-Based Study. Front Immunol 2019; 10: 1428.

Wu Pingping, Cai Wenqin, Lin Xiaozhen. A case of POEMS syndrome with numbness and proteinuria in both lower limbs. Chinese Journal of Hypertension 2020; 28: 1229-1233.

Yang Guangwei and Deng Nan. Clinical efficacy of methylprednisolone combined with gamma globulin in the treatment of acute Guillain-Barre syndrome. Journal of Clinical Medicine 2019; A1: 69-70. 